TRANSACTIONS OF THE

AMERICAN MATHEMATICAL SOCIETY

Volume 184, October 1973

\title{
RESTRICTING A SCHAUDER BASIS TO A SET OF POSITIVE MEASURE
}

\author{
BY
}

\section{JAMES SHIREY}

ABSTRACT. Let $\left\{f_{n}\right\}$ be an orthonormal system of functions on $[0,1]$ containing a subsystem $\left\{f_{n_{k}}\right\}$ for which (a) $f_{n_{k}} \rightarrow 0$ weakly. in $L_{2}$, and (b) given $E \subset[0,1],|E|>0$, Lim Inf $\int_{E}\left|f_{n_{k}}(x)\right| d x>0$. There then exists a subsystem $\left\{g_{n}\right\}$ of $\left\{f_{n}\right\}$ such that for any set $E$ as above, the linear span of $\left\{g_{n}\right\}$ in $L_{1}(E)$ is not dense.

For every set $E$ as above, there is an element of $L_{p}(E), 1<p<\infty$, whose Walsh series expansion converges conditionally and an element of $L_{1}(E)$ whose Haar series expansion converges conditionally.

1. Gaposhkin, in a discussion of certa in properties of "lacunary" systems of functions, see [G], makes extensive use of the following notion: A sequence $\left\{f_{n}\right\}$ of real-valued functions on $[0,1]$ is a "Riesz system" if the following estimates hold:

$$
A_{1}\left(\sum_{1}^{N} C_{n}^{2}\right)^{1 / 2} \leq \int_{0}^{1}\left|\sum_{1}^{N} C_{n} f_{n}(x)\right| d x \leq A_{2}\left(\sum_{1}^{N} C_{n}^{2}\right)^{1 / 2}, \quad N \geq 1,
$$

where the constants $0<A_{1} \leq A_{2}$ are independent of the choice of $\left\{C_{n}\right\}$ and of $N$. $A_{n}$ inequality of this type is used as a definition of the term "lacunary" in [KP] in an examination of complemented subspaces of the $L_{p}$ spaces. Moreover, the classical definition of a lacunary trigonometric system is used primarily to insure that such an inequality obtains [ $\left.\mathrm{Z}_{\mathrm{y}}, \mathrm{p} .203\right]$. One difficulty in the application of this notion is finding enough Riesz systems in context. To this end, a sequence $\left\{f_{n}\right\}$ is said to have "property (B)" if there is a subsequence $\left\{f_{n_{k}}\right\}$ such that $f_{n_{k}} \rightarrow 0$ weakly in $L_{2}$ and $\operatorname{Lim} \operatorname{Inf} \int_{0}^{1}\left|f_{n_{k}}(x)\right| d x>0$.

A sequence $\left\{f_{n}\right\}$ is said to have "property $\left(B^{\prime}\right)$ " if there is a subsequence $\left\{f_{n_{k}}\right\}$ such that $f_{n_{k}} \rightarrow 0$ weakly in $L_{2}$ and $\operatorname{Lim} \operatorname{Inf} \int_{E}\left|f_{n_{k}}(x)\right| d x>0$ whenever $E \subset(0,1)$ and $|E|^{n}>0$. The Lemma 1.2.6 of [G] shows that any sequence of functions having property $(B)$ contains a subsequence that is a Riesz system. Lemma $1.2 .6^{\prime}$ of $[G]$ shows that for any sequence of functions $\left\{f_{n}\right\}$ having property $\left(B^{\prime}\right)$ there is a subsequence $\left\{f_{n_{k}}\right\}$ for which the following obtains: given $E C$ $[0,1]$ and $|E|>0$, there is a $k_{0}=k_{0}(E)$ such that $\left\{f_{n_{k}}\right\}_{k>k_{0}}$ is a Riesz system when the $f_{n_{k}}$ are restricted to the set $E$.

Received by the editors October 23, 1972.

AMS (MOS) subject classifications (1970). Primary 42A64, 42A44.

Copyright $\odot$ 1974, American Mathematical Society 


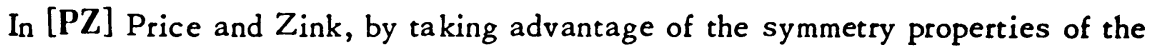
Rademacher functions, conclude that for any set $E \subset(0,1),|E|>0$, the closed linear span of the Rademacher functions in $L_{2}(E)$ is not all of $L_{2}(E)$. We offer another explanation for this phenomenon by noting that the conclusion of Lemma 1.2.6' (cited above) applies to the Rademacher system. We also obtain a generalized form of the Khintchine inequality [K, p. 130] that is of use in the next section.

Let $\|f\|=\int_{0}^{1}|f(t)| d t$ and $\|f\|_{E}=\int_{E}|f(t)| d t$ when $|E|>0$. For each $f$ in $L_{1}(0,1)$ define

$$
f^{E}(t)= \begin{cases}f(t) & \text { if } t \text { is in } E, \\ 0 & \text { otherwise. }\end{cases}
$$

For $X \subset L_{1}(0,1)$ let $X^{E}$ denote the closure in $L_{1}(E)$ of $\left\{f^{E}: f\right.$ is in $\left.x\right\}$.

A set $S \subset L_{1}(0,1)$ is "complete on the set $E$ " where $|E|>0$ provided that $S^{E}=L_{1}(E)$. Otherwise, $S$ is "incomplete on the set $E . "$

Lemma 1. Let $X$ be a subspace of $L_{1}(0,1)$ baving a separable topological dual $X^{\prime}$ and for which $X^{E}$ bas finite codimension in $L_{1}(E),|E|>0$.

There then exists an $f$ in $X^{E}$ such that if $\left\{f_{n}\right\} \subset X$ and $\left\|f-f_{n}\right\|_{E}=o(1)$, then $\left\|f_{n}\right\| \rightarrow \infty$. Such functions comprise all of $X^{E}$ except for a set of the first category in $X^{E}$.

Proof. There can be no constant $M>0$ such that given an $\epsilon>0$ and an $f$ in $X^{E}$, there exists a $g$ in $X$ for which $\|f-g\|_{E}$ and $\|g\| \leq M\|f\|_{E}$, where $M$ is independent of $\epsilon$ and $f$. For, suppose that such an $M$ exists. Let

$$
R=\left\{f^{E}: f \in X,\|f\|_{E} \leq 1, \text { and }\|f\| \leq M\right\}, \quad S=\left\{f: f \in X^{E} \text {, and }\|f\|_{E} \leq 1\right\},
$$

and let $T \in\left(X^{E}\right)^{\prime}$. T can be extended to an element of $L_{1}^{\prime}(0,1)$, and so there exists a bounded measurable function $b$ for which $T(f)=\int_{E} f(t) b(t) d t$.

Let $\|T\|$ denote the norm of $T$ as an operator on $X^{E}$, and let $N(T)$ denote the norm of $T$ as an operater on $X$. Since $R$ is by assumption dense in $S$,

$$
M N(T) \geq \sup \{|T(f)|: f \in R\}=\sup \{|T(f)|: f \in S\}=\|T\| \text {. }
$$

The nonseparability of $\left(X^{E}\right)^{\prime}$ now implies the nonseparability of $X^{\prime}$, a contradiction.

Now let $K_{n}=\left\{f \in X^{E}:\|f\|_{E} \leq 1\right.$ and there is a sequence $\left\{f_{m}\right\} \subset X$ for which $\left\|f_{m}-f\right\|_{E}=o(1)$ and $\left\|f_{m}\right\| \leq n$, for all $\left.m\right\}$.

Each $K_{n}$ is closed, convex, symmetric with respect to the origin in $X^{E}$. From what has been shown above, $K_{n}$ is not all of the unit ball in $X^{E}$ and is consequently nowhere dense. Thus $\bigcup_{n} K_{n}$ is of the first category, which is precisely the conclusion desired. 
A set $S \subset L_{1}(0,1)$ is "complete on a set $E$ " where $|E|>0$ provided that for any $f$ in $L_{1}(0,1)$ and any $\epsilon>0$, there is a $g$ in $S$ for which $\|f-g\|_{E}<\epsilon$.

Theorem 2. Let $\left\{f_{n}\right\}$ be an orthonormal sequence of functions in $L_{1}(0,1)$, $E \subset(0,1)$, and $|E|>0$, and let $X$ denote the closed linear span of $\left\{f_{n}\right\}$ in $L_{1}(0,1)$.

If $X^{\prime}$ is separable and $\left\{f_{n}^{E}\right\}$ is a Riesz system, then $\left\{f_{n}\right\}$ is incomplete on the set $E$.

Proof. Suppose $\left\{f_{n}\right\}$ is complete on the set $E$. Let $f$ be as in the conclusion of Lemma 1 and let $\left\{g_{n}\right\} \subset X$ be such that $\left\|f-g_{n}\right\|_{E}=o(1)$. Lemma 1 implies that $\left\|g_{n}\right\| \rightarrow \infty$ but the hypothesized properties of $\left\{f_{n}\right\}$ imply that $\left\{\left\|g_{n}\right\|\right\}$ is bounded, a contradiction.

Corollary 3. Every orthonormal system $\left\{f_{n}\right\}$ satisfying property $\left(\mathrm{B}^{\prime}\right)$ contains a subsystem that is incomplete on every set of positive measure.

Proof. Lemma 1.2.6 of [G] assures the existence of a subsystem $\left\{f_{n_{k}}\right\}$ having the following property. Given $E \subset(0,1),|E|>0$, there is a $k_{0}=k_{0}(E)$ such that $\left\{f_{n_{k}}\right\}_{k>k_{0}}$ and $\left\{f_{n_{k}}^{E}\right\}_{k>k_{0}}$ are both Riesz systems. Theorem 2 now implies that $\left\{f_{n_{k}}\right\}$ is incomplete on the set $E$, proving the corollary.

As a special case, we consider the Walsh system [K, p. 132]. This system is easily seen to satisfy property $\left(B^{\prime}\right)$.

Lemma 4. Let $\left\{w_{n}\right\}$ be a subsystem of the orthonormal system of Walsh pos. sessing the property $(*)$ :

(*) if $b_{1}, \ldots, b_{k}, i_{1}, \ldots, i_{k}$ denote positive integers, then

$$
\int_{0}^{1} w_{i_{1}}^{b_{1}}(t) \cdots w_{i_{k}}^{b_{k}}(t) d t= \begin{cases}1, & \text { when all } b_{i} \text { are even, } \\ 0, & \text { otherwise. }\end{cases}
$$

Let $1 \leq p \leq 2, E \subset[0,1],|E|>0,0<\epsilon<|E| / 5$, and let $K \subset E$ with $|E \sim K|$ $<\epsilon$. Then there are constants $A, B$, and $N$ depending only on $E$ sucb that for any $n>m>N$, and any sequence $a_{m} \cdots a_{n}$ of real numbers

$$
A\left(\sum_{m}^{n} a_{i}^{2}\right)^{1 / 2} \leq\left(\int_{K}\left|\sum_{m}^{n} a_{i} w_{i}(t)\right|^{p} d t\right)^{1 / p} \leq B\left(\sum_{m}^{n} a_{i}^{2}\right)^{1 / 2} .
$$

Proof. For any measurable set $S \subset[0,1]$, define $S_{i j} \equiv \int_{S} w_{i}(t) w_{j}(t) d t$. Note that $\left\{w_{i} w_{j}\right\}_{i<j}$ is a subsystem of the Walsh system and that $S_{i j}$ is the $i$, $j$ th Walsh-Fourier coefficient of the characteristic function of $S$. Bessel's inequality implies that $\Sigma_{i<j} s_{i j}^{2} \leq|S|$. 
Since $\|\cdot\|_{1} \leq\|\cdot\|_{p} \leq\|\cdot\|_{2}$, it is sufficient to establish the conclusion of the lemma for $p=1$ and $p=2$. For $p=2$

$$
\begin{aligned}
\int_{K}\left(\sum_{m}^{n} a_{i} w_{i}(t)\right)^{2} d t & =\int_{K}\left(\sum_{m}^{n} a_{i}^{2}+2 \sum_{m \leq i<j \leq n} a_{i} a_{j} w_{i}(t) w_{j}(t)\right) d t \\
& \leq|K| \sum_{m}^{n} a_{i}^{2}+2\left(\sum_{m \leq i<j \leq n} a_{i}^{2} a_{j}^{2}\right)^{1 / 2}\left(\sum_{m \leq i<j \leq n} K_{i j}^{2}\right)^{1 / 2} .
\end{aligned}
$$

A direct calculation yields $\left(\sum_{m \leq i<j \leq n} a_{i}^{2} a_{j}^{2}\right)^{1 / 2}=\sum_{m}^{n} a_{i}^{2}$. Also,

$$
\begin{aligned}
\left(\sum_{m \leq i<j \leq n .} K_{i j}^{2}\right)^{1 / 2} & \leq\left(\sum_{m \leq i<j \leq n} E_{i j}^{2}\right)^{1 / 2}+\left(\sum_{m \leq i<j \leq n}(E \sim K)_{i j}^{2}\right)^{1 / 2} \\
& \leq\left(\sum_{m \leq i<j \leq n} E_{i j}^{2}\right)^{1 / 2}+\epsilon_{0}
\end{aligned}
$$

Thus, if $N$ is chosen so that $\Sigma_{N}^{\infty} E_{i j}^{2}<\epsilon$, and $n>m>N$, then

$$
\begin{aligned}
\int_{K}\left(\sum_{m}^{n} a_{i} w_{i}(t)\right)^{2} d t & \leq|E| \sum_{m}^{n} a_{i}^{2}+2\left(\sum_{m}^{n} a_{i}^{2}\right)(2 \epsilon) \\
& =(|E|+4 \epsilon)\left(\sum_{m}^{n} a_{i}^{2}\right)
\end{aligned}
$$

Similar considerations will establish the other half of the desired inequality. One obtains

$$
\int_{K}\left(\sum_{m}^{n} a_{i} w_{i}(t)\right)^{2} d t \geq(|E|-5 \epsilon)\left(\sum_{m}^{n} a_{i}^{2}\right),
$$

and the case $p=2$ is established.

For $p=1$

$$
\begin{aligned}
\int_{K}\left|\sum_{m}^{n} a_{i} w_{i}(t)\right| d t & \leq|E|^{1 / 2}\left(\int_{K}\left(\sum_{m}^{n} a_{i} w_{i}(t)\right)^{2} d t\right)^{1 / 2} \\
& \leq|E|^{1 / 2}(|E|+4 \epsilon)^{1 / 2}\left(\sum_{m}^{n} a_{i}^{2}\right)^{1 / 2}
\end{aligned}
$$

provided that $n>m>N(\epsilon)$.

For the left-hand side we have: 


$$
\begin{aligned}
\left(\sum_{m}^{n} a_{k}^{2}\right)^{1 / 2} & \leq(|E|-5 \epsilon)^{-1 / 2}\left(\int_{K}\left(\sum_{m}^{n} a_{i} w_{i}(t)\right)^{2} d t\right)^{1 / 2} \\
& =A\left(\int_{K}\left(\sum_{m}^{n} a_{i} w_{i}(t)\right)^{2 / 3}\left(\sum_{m}^{n} a_{i} w_{i}(t)\right)^{4 / 3} d t\right)^{1 / 2} .
\end{aligned}
$$

An application of Hölder's inequality with $p=3 / 2$ and $q=3$ yields

$$
\left(\sum_{m}^{n} a_{k}^{2}\right)^{1 / 2} \leq A\left(\int_{K}\left|\sum_{m}^{n} a_{i} w_{i}(t)\right| d t\right)^{1 / 3}\left(\int_{K}\left(\sum_{m}^{n} a_{i} w_{i}(t)\right)^{4} d t\right)^{1 / 6} .
$$

Observe that the Khintchine inequality $[K$, p. 130] is valid for any system of Walsh functions which possesses property $(*)$. Taking this into account, set $p=$ 4 in the Khintchine inequality. The last term in line $\left(_{+}\right)$above can now be replaced by $B\left(\Sigma_{m}^{n} a_{i}\right)^{1 / 3}$, where $B>0$ depends only on the choice of $p=4$, without disturbing the sense of the inequality. The desired result is obtained when both sides of the resultant expression are cubed.

Corollary 5. No system $\left\{w_{k}\right\}$ of Walsb functions possessing property (*) of Lemma 4 can be complete on any set of positive measure.

Proof. Let $E \subset(0,1),|E|>0$. Lemma 4 implies the existence of $N=N(E)$ for which $\left\{w_{n}\right\}_{n>N}$ and $\left\{w_{n}^{E}\right\}_{n>N}$ are both Riesz systems. Theorem 2 now implies that $\left\{w_{n}\right\}$ is incomplete on the set $E$.

A "quasi-basis" for a Banach space $(X,\|\cdot\|)$ is a double sequence $\left\{x_{n}, X_{n}\right\}$ of elements of $X$ and of continuous linear functionals, respectively, such that the series $\Sigma_{1}^{\infty} X_{n}(x)_{n}$ converges in norm to $x$ for each $x$ in $X$. Such a structure may arise in the following way.

Suppose $\left\{y_{n}, Y_{n}\right\}$ is a Schauder basis for $L_{p}(0,1), 1 \leq p<\infty$. Let $|E|>0$ and define for all $z$ in $L_{p}(0,1)$

$$
z^{E}(t)= \begin{cases}z(t), & t \text { in } E \\ 0, & \text { otherwise. }\end{cases}
$$

For each given $\phi$ in $L_{p}^{\prime}(0,1)$, define $\phi^{E}(z)=\phi\left(z^{E}\right)$ for any $z$ in $L_{p}(0,1)$.

It is easily verified that $\left\{y_{n}^{E}, Y_{n}^{E}\right\}$ is a quasi-basis for $L_{p}(E)$. This double sequence shall be called the "restriction of $\left\{y_{n}, Y_{n}\right\}$ to the set $E$."

The quasi-basis $\left\{x_{n}, X_{n}\right\}$ is "unconditional" if $\Sigma_{1}^{\infty} X_{n}(x) x_{n}$ converges unconditionally for every $x$ in $X$. Otherwise, the quasi-basis is "conditional." The restriction of an unconditional basis for $L_{p}(0,1)$ to any set $E,|E|>0$, is clearly an unconditional quasi-basis for $L_{p}(E)$. A problem that arises in this context is the determination of the sets $E$, if there are any, for which a given conditional basis for $L_{p}(0,1)$ restricts to an unconditional quasi-basis for 
$L_{p}(E)$. Two special cases are considered below: it turns out that no such sets exist for the Walsh system $[K, p .132]$ in $L_{p}(0,1), 1<p<\infty$, or for the Haar system $\left[K\right.$, p. 120] in $L_{1}(0,1)$.

Let $(X,\|\cdot\|)$ denote a Banach space of equivalence classes of Lebesgue measurable functions on $[0,1]$ (see $[\mathrm{L}$, p. 66] and $[\mathrm{Z}$, Chapter 15]). Such spaces may be defined by specifying a certain class $H$ of elements of $L_{1}(0,1)$ and defining

$$
\|x\|=\sup \left\{\int_{0}^{1}|x(t) b(t)| d t: b \in H\right\} \text { and } X=\{x:\|x\|<\infty\} \text {. }
$$

Various conditions may be imposed on $H$ in order that $(X,\|\cdot\|)$ turns out as a Banach space.

Theorem 6. Given a quasi-basis $\left\{x_{n}, X_{n}\right\}$ for $(X,\|\cdot\|)$. Let $\left\{r_{n}\right\}$. denote the Rademacher system and define

$$
C=\left\{\theta: \sum_{1}^{\infty} r_{k}(\theta) X_{k}(x) x_{k} \text { converges for all } x \text { in } X\right\} \text {. }
$$

Let $Z_{N}=\left\{x \in X: X_{1}(x)=\cdots=X_{N}(x)=0\right\}$ and let $G(x)=\left\|\left(\Sigma_{1}^{\infty} X_{n}^{2}(x) x_{n}^{2}\right)^{1 / 2}\right\|$.

Then $C$ is a Borel set, and if $|C|>0$ there is an $N$ for which the identity mapping $\left(Z_{N},\|\cdot\|\right) \rightarrow\left(Z_{N}, G(\cdot)\right)$ is continuous.

Proof. Let $x(n, m, \theta, t)=\Sigma_{n}^{m} r_{k}(\theta) X_{k}(x) x_{k}(t)$. For each $(n, m)$, $\|x(n, m, \theta, \cdot)\|$ is a step function of $\theta$ and

$$
C=\{\theta: \underset{n, m \rightarrow \infty}{\operatorname{Lim} \sup }\|x(n, m, \theta, \cdot)\|=0\}
$$

$C$ is therefore a Borel set.

For each $\theta$ in $C$, the sequence of linear operaters $T_{n \theta}(\cdot)$ defined by

$$
T_{n \theta}(x)=\sum_{1}^{n} r_{k}(\theta) X_{k}(x) x_{k}
$$

is uniformly bounded by virtue of the Banach-Steinhaus theorem. The set $S_{M} \equiv$ $\left\{\theta:\left\|T_{n \theta}\right\| \leq M\right.$ for all $\left.n\right\}$ is clearly a Borel set, and $U\left(s_{M} \cap C\right)=C$. Since $|C|>0$, there is a constant $K>0$ for which $\left|S_{K} \cap C\right|>0$. Define $S \equiv S_{K} \cap C$, and for each $\theta$ in $S$ let $T_{\theta}(x) \equiv \lim _{n} T_{n \theta}(x)$. Then,

$$
\left\|T_{\theta}(x)\right\|=\lim _{n}\left\|T_{n \theta}(x)\right\| \leq M\|x\| \quad \text { for all } \theta \in S,
$$

and $S$ is seen to be a Borel set of positive measure 
For any $\epsilon>0$ there is, by definition of $\|\cdot\|$, an $b$ in $H$ such that

$$
G(x) \leq \int_{0}^{1}\left(\sum_{1}^{\infty} x_{k}^{2}(x) x_{k}^{2}(t)\right)^{1 / 2}|b(t)| d t+\epsilon .
$$

By Lemma 4, if $F \subset S$ is of sufficiently large measure, there is a $B>0$ and an integer $N>0$, depending only on $S$, such that

$$
B\left(\sum_{N}^{n} X_{k}^{2}(x) x_{k}^{2}(t)\right)^{1 / 2}|b(t)| \leq \int_{F}|x(N, n, \theta, t) b(t)| d \theta
$$

for every $x$ in $X$, and for every $n>N$.

For each $\theta$ in $S$,

$$
\operatorname{Lim}_{n} \int_{0}^{1}|x(N, n, \theta, t) b(t)| d t=\int_{0}^{1}|x(n, \infty, \theta, t) b(t)| d t .
$$

By Egoroff's theorem there is $F C S$ in which the convergence in (4) is uniform, and having measure large enough so that line (3) obtains. We may integrate (4) with respect to $\theta$ over the set $F$, and the order of integration on the left side may be reversed. This yields

(5) $\operatorname{Lim}_{n} \int_{0}^{1}\left[\int_{F}|x(N, n, \theta, t) b(t)| d \theta\right] d t=\int_{F}\left[\int_{0}^{1}|x(N, \infty, \theta, t) b(t)| d t\right] d \theta$.

Assume now that $x$ is in $Z_{N}$, and combine (1), (2), (3), and (5):

$$
G(x) \leq \frac{1}{B} \int_{F}\left[\int_{0}^{1}|x(N, \infty, \theta, t) b(t)| d t\right] d \theta+\epsilon \leq \frac{1}{B} \int_{F}\left\|T_{\theta}(x)\right\| d \theta+\epsilon \leq \frac{M}{B}\|x\|+\epsilon
$$

for all $x$ in $Z_{N}$, and for any $\epsilon>0$.

The norm $G($.$) and unconditional convergence have been discussed previously$ in [0], and in the case that $\left\{x_{n}, X_{n}\right\}$ is an unconditional basis it has been shown in $[\mathrm{SZ}]$ that the norms $G(\cdot)$ and $\|\cdot\|$ are equivalent.

Theorem 6 can be used to partially extend Lemma 4 to all of the spaces $L_{p}(0,1), 1<p<\infty$ :

Corollary 7. Let $\left\{w_{n}\right\}$ be a subsystem of the Walsh system possessing the property $(*)$ described in Lemma 4 , and let $1 \leq p<\infty,|E|>0$. Then there are constants $A, B$, and $N$ depending only on $E$ sucb that for any $n>m>N$ and any sequence $a_{m} \cdots a_{n}$ of real numbers

$$
A\left(\sum_{m}^{n} a_{i}^{2}\right)^{1 / 2} \leq\left(\int_{E}\left|\sum_{m}^{n} a_{i} w_{i}(t)\right|^{p} d t\right)^{1 / p} \leq B\left(\sum_{m}^{n} a_{i}^{2}\right)^{1 / 2}
$$


Proof. For $2 \leq p<\infty$, let $N$ be determined by the set $E$ as in Lemma 4 , and let $Y$ be the closed linear span of $\left\{w_{i}^{E}\right\}_{i \geq N}$ in $L_{p}(E)$. If continuous linear functionals $W_{i}$ on $Y$ can be found for which $\left\{w_{i}^{E}, W_{i}\right\}_{i \geq N}$ is an unconditional basis for $Y$, the desired conclusion will then follow from Lemma 4, Theorem 6 and the fact that $\|\cdot\|_{p} \geq\|\cdot\|_{2}$.

Lemma 4 implies that for each $x$ in $Y$, there is a unique sequence $\left\{a_{i}\right\}$ in $l_{2}$ for which the series $\Sigma_{i \geq N} a_{i} w_{i}^{E}$ converges unconditionally to $x$ in the norm of $L_{2}(0,1)$. The Khintchine inequality with the functions $w_{i}$ substituted for Rademacher's functions [ see proof of Lemma 4 above] implies that $\Sigma_{i \geq N} a_{i} w_{i} \equiv$ $\bar{x}$ converges unconditionally in the norm of $L_{p}(0,1)$ and that the mapping $x \rightarrow \bar{x}$ taking $Y \rightarrow L_{p}(0,1)$ is continuous in that norm.

Clearly, the functionals $W_{i}$ defined by $W_{i}(x) \equiv \int_{0}^{1} w_{i}(t) \bar{x}(t) d t$ are as desired.

The case $1 \leq p \leq 2$ is a special case of Lemma 1 , proving the corollary.

It is known that the Walsh system is a conditional basis for $L_{p}(0,1), 1<p$ $<\infty, p \neq 2,[0]$ and $[P]$. Also, the Haar system is a conditional basis for $L_{1}(0,1)$, $[D]$ and $[M]$. The conditionality of these systems is preserved under restriction to a set of positive measure.

Theorem 8. Let $\left\{w_{n}, W_{n}\right\}$ denote the quasi-basis for $L_{p}(E), 1<p<\infty$, ob. tained by restricting the Walsh system to a set $E,|E|>0$.

If $p \neq 2$, then $\left\{w_{n}, W_{n}\right\}$ is a conditional quasi-basis for $L_{p}(E)$. If $p=2$, then this quasi-bas is is unconditional.

Proof. If $p=2$, the Walsh system is an unconditional basis for $L_{p}(0,1)$, and so $\left\{w_{n}, W_{n}\right\}$ is an unconditional quasi-basis for $L_{p}(E)$.

The remaining cases $1<p<2$ and $2<p<\infty$ are considered separately. For $p<2$, let $x \in L_{p}(E) \sim L_{2}(E)$. Then

$$
G(x)=\left\|\left(\sum_{1}^{\infty} W_{n}^{2}(x) w_{n}^{2}\right)^{1 / 2}\right\|_{p}=\left(\sum_{1}^{\infty} W_{n}^{2}(x)\right)^{1 / 2}|E|=\infty .
$$

It follows that the series $\Sigma_{1}^{\infty} W_{n}(x) w_{n}$ is conditionally convergent [0].

For $p>2$, let $y \in L_{q}(E)$ where $1 / p+1 / q=1$. Were $\left\{w_{n}, W_{n}\right\}$ an unconditional quasi-basis for $L_{p}(E)$, then

$$
\epsilon(x) \equiv \operatorname{Lim}_{n} \sum_{1}^{n} W_{k}(x) \epsilon_{k} w_{k}
$$

would exist for every $x$ in $L_{p}(E)$ and for every sequence $\epsilon \equiv\left\{\epsilon_{k}\right\}$ where $\epsilon_{k}= \pm 1$. Then 


$$
(y, \epsilon(x))=\sum_{1}^{\infty} W_{k}(x) \epsilon_{k}\left(w_{k}, y\right)=\sum_{1}^{\infty} W_{k}(y) \epsilon_{k}\left(w_{k}, x\right)
$$

would converge for all $x$ and $\epsilon$.

The series $\Sigma_{1}^{\infty} W_{k}(y) w_{k}$ would then be subseries convergent in the weak topology on $L_{q}(E)$ and hence subseries convergent in the norm topology [D, p. 60]. $\left\{w_{k}, W_{k}\right\}$ would then be an unconditional quasi-basis for $L_{q}(E)$, which is impossible since $1<q<2$.

Theorem 9. Let $\left\{b_{n p}^{E}, H_{n p}^{E}\right\}$ denote the quasi-basis for $L_{1}(E)$ obtained by re. stricting the Haar system to a set $E,|E|>0$.

Then this system is a conditional quasi-basis for $L_{1}(E)$.

Proof. Theorem 6 implies that if $\left\{b_{n p}^{E}, H_{n p}^{E}\right\}$ were an unconditional quasibasis for $L_{1}(E)$, then the norm $\|\cdot\|$ would dominate the norm $G(\cdot)$ on some space $Z_{N}=\left\{x \in L_{1}(E): H_{n p}(x)=0\right.$ for $\left.n \leq N, p=0,1, \cdots, 2^{n}-1\right\}$. But this cannot be the case: a sequence $\left\{y_{p}\right\}$ is constructed for which

(A) $\left\{y_{p}\right\} \subset Z_{N}$,

(B) $\left\{y_{p}\right\}$ is bounded in $\left(L_{1}(E),\|\cdot\|\right)$,

(C) $\left\{y_{p}\right\}$ is unbounded in $\left(L_{1}(E), G(\cdot)\right)$.

Let $p$ be a fixed positive integer, $p>4$, and let $\epsilon<1 / p$. Since almost every point of $E$ is a point of metric density 1 , there exists a sequence $\left\{I_{k}\right\}_{k=0}^{p}$ of dyadic intervals, each of which is the support of a Haar function which shall be denoted by $b_{k}$, and for which

$I_{k+1}$ is the left half of $I_{k}$,

$\left|I_{k}\right|=2^{-n-k}, k=0,1, \cdots, p$,

$0 \leq 1-\left|E \cap I_{k}\right| /\left|I_{k}\right|<\epsilon, k=0, \cdots, p$.

Let $E_{k}=E \cap I_{k}$ and split $E_{k}$ into a left half $L_{k}$ and a right half $R_{k}$ of equal measure. Define, for $k=0,1, \cdots, p$,

$$
Y_{k}(t)= \begin{cases}\sqrt{ } 2^{n+k} & \text { if } t \text { is in } L_{k}, \\ -\sqrt{ } 2^{n+k} & \text { if } t \text { is in } R_{k}, \\ 0 & \text { otherwise, }\end{cases}
$$

and let $y_{p}=\sum_{k=0}^{p} \sqrt{2^{n+k}} Y_{k}$.

The proof shall be completed when it is demonstrated that the sequence $\left\{y_{p}\right\}$ possesses properties (A), (B) and (C) above. This necessitates estimates on $H_{i}^{E}\left(y_{p}\right)=\sum_{k=0}^{p} \sqrt{ } 2^{n+k} H_{i}\left(Y_{k}\right)$.

The function $Y_{k}$ resembles the Haar function $b_{k}$ restricted to the set $E$. In particular, if $e_{k}$ is defined by the formula

$$
Y_{k}(t)=b_{k}^{E}(t)+e_{k}(t)
$$


then

$$
\begin{aligned}
\left|\operatorname{supp} e_{k}\right| & \leq 1 / 2\left(\left|I_{k}\right|-\left|E_{k}\right|\right) \leq \epsilon 2^{-n-k-1}, \\
\left|e_{k}(t)\right| & \leq 2 \sqrt{ } 2^{n+k} .
\end{aligned}
$$

Estimation of $H_{i}^{E}\left(Y_{k}\right)$ for $k \geq i$ :

$$
\left|H_{i}^{E}\left(b_{i}\right)\right|=2^{n+i}\left|E_{i}\right|
$$

and

$$
\left|H_{i}^{E}\left(e_{i}\right)\right| \leq \sqrt{2^{n+i}} \max \left|e_{i}(t)\right|\left|\operatorname{supp} e_{i}\right|=2^{n+i+1}\left|\operatorname{supp} e_{i}\right| \leq \epsilon
$$

Thus,

$$
\left|H_{i}^{E}\left(Y_{i}\right)\right| \geq 2^{n+i}\left|E_{i}\right|-\epsilon \geq 1-3 \epsilon .
$$

If $k>i$, then supp $Y_{k}$ is contained in that part of $E_{i}$ on which $b_{i}$ is of constant sign. In this case, $H_{i}^{E}\left(Y_{k}\right)=0$.

Estimation of $H_{i}^{E}\left(Y_{k}\right)$ for $k<i$ :

$$
\begin{aligned}
& \left|H_{i}^{E}\left(b_{k}\right)\right| \leq \sqrt{ } 2^{n+i} \sqrt{2} 2^{n+k}\left(\left|I_{i}\right|-\left|E_{i}\right|\right) \leq \epsilon \sqrt{ } 2^{n+i} \sqrt{ } 2^{n+k} 2^{-n-i}=\epsilon \sqrt{ } 2^{k-i} \\
& \left|H_{i}^{E}\left(e_{k}\right)\right| \leq 2 \sqrt{ } 2^{n+i} \sqrt{ } 2^{n+k}\left|\operatorname{supp} e_{k} \cap E_{i}\right| .
\end{aligned}
$$

If $k<i-1, \operatorname{supp} e_{k} \cap E_{i}=\varnothing$ and then $H_{i}^{E}\left(e_{k}\right)=0$. Otherwise, $\left|H_{i}^{E}\left(e_{i-1}\right)\right| \leq$ $2 \sqrt{ } 2^{n+i} \sqrt{ } 2^{n+i-1} 2^{-n-i} \epsilon=\epsilon \sqrt{ } 2$. This gives

$$
\begin{aligned}
\left|H_{i}^{E}\left(Y_{k}\right)\right| & \leq \epsilon \sqrt{ } 2^{k-i} \quad \text { if } k<i-1, \\
\left|H_{i}^{E}\left(Y_{i-1}\right)\right| & \leq \epsilon \sqrt{ } 2+\epsilon / \sqrt{ } 2 \leq 3 \epsilon .
\end{aligned}
$$

Upon combining the above estimates in $H_{i}^{E}\left(Y_{k}\right)$,

$$
\begin{aligned}
& \left|H_{i}^{E}\left(y_{p}\right)\right|=\left|\sum_{k=0}^{p} \sqrt{ } 2^{n+k} H_{i}^{E}\left(Y_{k}\right)\right| \\
& \geq\left|\sqrt{ } 2^{n+i} H_{i}^{E}\left(Y_{i}\right)\right|-\sqrt{ } 2^{n+i-1}\left|H_{i}^{E}\left(Y_{i-1}\right)\right| \quad \sum_{k=0}^{i-2} \sqrt{ } 2^{n+k}\left|H_{i}^{E}\left(Y_{k}\right)\right| \\
& \therefore \sqrt{2} 2^{n+i}(1-3 \epsilon)-3 \epsilon \sqrt{2^{n+i-1}}-\epsilon \sum_{k=0}^{i-2} \sqrt{2^{n+k}} \sqrt{2^{k-i}} \\
& \geq \sqrt{ } 2^{n+i}(1-6 \epsilon)-\epsilon \sqrt{2^{n-i}} \sum_{k=0}^{i-2} 2^{k} \\
& \geq \sqrt{ } 2^{n+i}(1-6 \epsilon)-\epsilon \sqrt{2^{n+i}}=\sqrt{ } 2^{n+i}(1-7 \epsilon) .
\end{aligned}
$$


Let $\|\cdot\|_{E}$ denote the $L_{1}$-norm of functions restricted to $E$. An application of the estimate above then yields

$$
\begin{aligned}
G\left(y_{p}\right) & \geq\left\|\left(\sum_{i=0}^{p}\left[H_{i}^{E}\left(Y_{p}\right)\right]^{2} b_{i}^{2}\right)^{1 / 2}\right\|_{E} \geq A\left\|\left(\sum_{i=0}^{p} 2^{n+i} b_{i}^{2}\right)^{1 / 2}\right\|_{E} \\
& \geq B \sum_{i=0}^{p} 2^{-n-i}\left(\sum_{k=0}^{i} 2^{2(n+k)}\right)^{1 / 2} \geq C_{p} .
\end{aligned}
$$

Statement (C) is thereby established. Moreover,

$$
\left\|y_{p}\right\| \leq\left\|\sum_{k=0}^{p} \sqrt{2^{n+k}} b_{k}\right\|+\left\|\sum_{k=0}^{p} \sqrt{2^{n+k}} e_{k}\right\| \leq 2+\sum_{k=0}^{p} \sqrt{2^{n+k}}\left\|e_{k}\right\| \leq 2+p \epsilon \leq 3 .
$$

This establishes statement (B). Given any $N$, it is clear that the intervals $I_{k}$ can be so chosen that the functions $y_{k}$ are all elements of $Z_{N}$. Then $y_{p}$ will also be an element of $Z_{N}$, and (A) is established.

\section{REFERENCES}

[D] M. M. Day, Normed linear spaces, 2nd rev. ed., Ergebnisse der Mathematik und ihrer Grenzgebiete, N. F., Heft 21, Academic Press, New York; Springer-Verlag, Berlin, 1962. MR $26 \# 2847$.

[G] V. F. Gapoškin, Lacunary series and independent functions, Uspehi Mat. Nauk 21 (1966), no. 6 (132), 3-82 = Russian Math. Surveys 21 (1966), no. 6, 1-82. MR 34 \#6374.

[K] S. Kaczmarz and H. Steinhaus, Theorie der Orthogonalreihen, reprint, Chelsea, New York, 1951. MR $20 \# 1148$.

[KP] M. I. Kadec and A. Pelczyński, Bases, lacunary sequences, and complemented subspaces in the spaces $L_{p}$, Studia Math. 21 (1961/62), 161-176. MR 27 \#2851.

[L] G. G. Lorentz, Bemstein polynomials, Mathematical Expositions, no. 8, Univ. of Toronto Press, Toronto, 1953. MR 15, 217.

[M] Jurg T. Marti, Introduction to the theory of bases, Springer-Verlag, New York, 1959.

[o] W. Orlicz, Über unbedingte Convergenz in Funktionräumen . I, Studia Math. 4 (1933), 33-37.

[P] R. E. A. C. Paley, A remarkable series of orthogonal functions, Proc. London Math. Soc. 34 (1931), 241-264.

[PZ] J. J. Price and R. E. Zink, On sets of completeness. for families of Haar functions, Trans. Amer. Math. Soc. 119 (1965), 262-269. MR 32 \#1499.

[SZ] J. Shirey and R. E. Zink, On unconditional bases in certain Banach function spaces, Studia Math. 36 (1970), 169-175. MR 43 \#899.

[z] A. C. Zaanen, An introduction to the theory of integration, rev. ed., North-Holland, Amsterdam; Interscience, New York, 1967. MR 36 \#5286.

[ $\mathrm{Zy}$ ] A. Zygmund, Trigonometrical series, 2 nd ed., reprinted with corrections and some additions, Cambridge Univ. Press, New York, 1968. MR 38 \#4882. 